\title{
Weinstein Gabor Transform and Applications
}

\author{
Hatem Mejjaoli ${ }^{1}$, Ahmedou Ould Ahmed Salem ${ }^{2}$ \\ ${ }^{1}$ Department of Mathematics, College of Sciences, King Faisal University, Ahsaa, KSA \\ ${ }^{2}$ Department of Mathematics, College of Education, King Khalid University, Mohayil, KSA \\ Email: hmejjaoli@kfu.edu.sa,ahmdoo@kku.edu.sa
}

Received January 12, 2012; revised February 28, 2012; accepted March 7, 2012

\begin{abstract}
In this paper we consider Weinstein operator. We define and study the continuous Gabor transform associated with this operator. We prove a Plancherel formula, an inversion formula and a weak uncertainty principle for it. As applications, we obtain analogous of Heisenberg's inequality for the generalized continuous Gabor transform. At the end we give the practical real inversion formula for the generalized continuous Gabor transform.
\end{abstract}

Keywords: Weinstein Gabor Transform; Practical Real Inversion Formula; Kernel Reproducing Theory

\section{Introduction}

We consider the Weinstein operator defined on $\left.\mathbb{R}^{d} \times\right] 0, \infty[$ by :

$$
\Delta_{\beta}:=\sum_{i=1}^{d+1} \frac{\partial^{2}}{\partial x_{i}^{2}}+\frac{2 \beta+1}{x_{d+1}} \frac{\partial}{\partial x_{d+1}}, \beta>-\frac{1}{2}=\Delta_{d}+\mathcal{L}_{\beta}
$$

where $\Delta_{d}$ is the Laplacian for the $d$-first variables and $\mathcal{L}_{\beta}$ the Bessel operator for the last variable, given by

$$
\mathcal{L}_{\beta}=\frac{\partial^{2}}{\partial x_{d+1}^{2}}+\frac{2 \beta+1}{x_{d+1}} \frac{\partial}{\partial x_{d+1}}, \beta>-\frac{1}{2} .
$$

For $d>2$, the operator $\Delta_{\beta}$ is the Laplace-Beltrami operator on the Riemanian space $\left.\mathbb{R}^{d} \times\right] 0, \infty[$ equipped with the metric

$$
\mathrm{d} s^{2}=x_{d+1}^{2(2 \beta+1) /(d-1)} \sum_{i=1}^{d+1} \mathrm{~d} x_{i}^{2}
$$

(cf. [1]).

The Weinstein operator $\Delta_{\beta}$ has several applications in pure and applied Mathematics especially in Fluid Mechanics (cf. [3]).

The harmonic analysis associated with the Weinstein operator is studied by Ben Nahia and Ben Salem (cf. $[1,2])$. In particular the authors have introduced and studied the generalized Fourier transform associated with the Weinstein operator. This transform is called the Weinstein transform. In this work we are interested to the Gabor transform associated with Weinstein operator.

Time-Frequency analysis plays a central role in signal analysis. Since years ago, it has been recognized that the global Fourier transform of a long time signal has a little practical value to method is preferred to the classical Fourier method, whenever the time dependence of the analyzed signal is of the same importance as its frequency dependence.

However, there exist strict limits to the maximal TimeFrequency resolution of this transform, similar to Heisenberg's uncertainty principles in the Fourier analysis.

In fact, Dennis Gabor [4] was the first to introduce the Gabor transform, in which he uses translations and modulations of a single Gaussian to represent one dimensional signal. Other names for this transform used in literature, are: short time Fourier transform, Weyl-Heisenberg transform, windowed Fourier transform.

In this paper, we are interested a generalized Gabor transform associated for the Weinstein transform. More precisely, we give here general reconstruction formulas and we give many applications. In the classical case the Gabor transform is very fundamental and has many applications to Mathematical Sciences.

The paper is organized as follows. In Section 2, we recall the main results about the harmonic analysis related to the Weinstein operator. In Section 3, we introduce the analogue of the continuous Gabor transform associated with the Weinstein operator and we give some harmonic properties for it (Plancheral formula, $L_{\beta}^{2}$ inverse formula, weak uncertainty for it). The Section 4 is devoted to prove the analogous of Heisenberg's inequality for the generalized continuous Gabor transform. In Section 5 using the kernel reproducing theory given by Saitoh [5] we study the problem of approximative concentration. In the last section we give a practical real inversion formulas and extremal function for the Weinstein-Gabor transform.

\section{Preliminaries}

In order to confirm the basic and standard notations we 
briefly overview the Weinstein operator and related harmonic analysis. Main references are [1,2].

In the following we denote by

$$
\begin{aligned}
& \mathbb{R}_{+}^{d+1}=\mathbb{R}^{d} \times[0, \infty[. \\
& \mathbb{R}_{+}^{2 d+2}:=\mathbb{R}_{+}^{d+1} \times \mathbb{R}_{+}^{d+1} . \\
& x=\left(x_{1}, \cdots, x_{d}, x_{d+1}\right)=\left(x^{\prime}, x_{d+1}\right) \in \mathbb{R}_{+}^{d+1} . \\
& B_{+}^{d+1}(0, n):=\left\{x \in \mathbb{R}_{+}^{d+1}:\|x\| \leq n\right\} .
\end{aligned}
$$

$C_{*}\left(\mathbb{R}^{d+1}\right)$ the space of continuous functions on $\mathbb{R}^{d+1}$, even with respect to the last variable.

$C_{*}^{p}\left(\mathbb{R}^{d+1}\right)$ the space of functions of class $C^{p}$ on $\mathbb{R}^{d+1}$, even with respect to the last variable.

$\mathcal{E}_{*}\left(\mathbb{R}^{d+1}\right)$ the space of $C^{\infty}$-functions on $\mathbb{R}^{d+1}$, even with respect to the last variable.

$\mathcal{S}_{*}\left(\mathbb{R}^{d+1}\right)$ the Schwartz space of rapidly decreasing functions on $\mathbb{R}^{d+1}$, even with respect to the last variable.

$D_{*}\left(\mathbb{R}^{d+1}\right)$ the space of $C^{\infty}$-functions on $\mathbb{R}^{d+1}$ which are of compact support, even with respect to the last variable.

We consider the Weinstein operator $\Delta_{\beta}$ defined by

$$
\begin{gathered}
\left.\forall x=\left(x^{\prime}, x_{d+1}\right) \in \mathbb{R}^{d} \times\right] 0, \infty[, \\
\Delta_{\beta} f(x)=\Delta_{x^{\prime}} f\left(x^{\prime}, x_{d+1}\right)+\mathcal{L}_{\beta, x_{d+1}} f\left(x^{\prime}, x_{d+1}\right), \\
f \in C_{*}^{2}\left(\mathbb{R}^{d+1}\right),
\end{gathered}
$$

where $\Delta_{x^{\prime}}$ is the Laplace operator on $\mathbb{R}^{d}$, and $\mathcal{L}_{\beta, x_{d+1}}$ the Bessel operator on $] 0, \infty[$ given by

$$
\mathcal{L}_{\beta, x_{d+1}}:=\frac{d^{2}}{\mathrm{~d} x_{d+1}^{2}}+\frac{2 \beta+1}{x_{d+1}} \frac{d}{\mathrm{~d} x_{d+1}}, \beta>-\frac{1}{2} .
$$

The Weinstein kernel $\Lambda$ is given by

$$
\begin{gathered}
\Lambda(x, z):=e^{i\left\langle x^{\prime}, z^{\prime}\right\rangle} j_{\beta}\left(x_{d+1} z_{d+1}\right), \\
\text { for all }(x, z) \in \mathbb{R}^{d+1} \times \mathbb{C}^{d+1},
\end{gathered}
$$

where $j_{\beta}\left(x_{d+1} z_{d+1}\right)$ is the normalized Bessel function. The Weinstein kernel satisfies the following properties:

1) For all $z, t \in \mathbb{C}^{d+1}$, we have

$$
\begin{aligned}
& \Lambda(z, t)=\Lambda(t, z) ; \Lambda(z, 0)=1 \text { and } \\
& \Lambda(\lambda z, t)=\Lambda(z, \lambda t), \text { for all } \lambda \in \mathbb{C} .
\end{aligned}
$$

2) For all $v \in \mathbb{N}^{d+1}, \quad x \in \mathbb{R}_{+}^{d+1}$ and $z \in \mathbb{C}^{d+1}$, we have

$$
\left|D_{z}^{v} \Lambda(x, z)\right| \leq\|x\|^{v \mid} \exp (\|x\|\|\operatorname{Im} z\|),
$$

where $\quad D_{z}^{v}=\frac{\partial^{v}}{\partial z_{1}^{v_{1}} \cdots \partial z_{d+1}^{v_{d+1}}}$ and $|v|=v_{1}+\cdots+v_{d+1}$. In particular

$$
|\Lambda(x, y)| \leq 1 \text {, for all } x, y \in \mathbb{R}_{+}^{d+1} .
$$

We denote by $L_{\beta}^{p}\left(\mathbb{R}_{+}^{d+1}\right)$ the space of measurable functions on $\mathbb{R}_{+}^{d+1}$ such that

$$
\begin{aligned}
\|f\|_{L_{\beta}^{p}\left(\mathbb{R}_{+}^{d+1}\right)}:=\left(\int_{\mathbb{R}_{+}^{d+1}}|f(x)|^{p} \mathrm{~d} \mu_{\beta}(x)\right)^{\frac{1}{p}}<\infty, \text { if } 1 \leq p<\infty, \\
\|f\|_{L_{\beta}^{\infty}\left(\mathbb{R}_{+}^{d+1}\right)}:=\operatorname{ess} \sup _{x \in \mathbb{R}_{+}^{d+1}}|f(x)|<\infty,
\end{aligned}
$$

where $\mathrm{d} \mu_{\beta}$ is the measure on $\mathbb{R}_{+}^{d+1}$ given by

$$
\mathrm{d} \mu_{\beta}\left(x^{\prime}, x_{d+1}\right):=\frac{x_{d+1}^{2 \beta+1}}{(2 \pi)^{\frac{d}{2}} 2^{\beta} \Gamma(\beta+1)} \mathrm{d} x .
$$
by

The Weinstein transform is given for $f$ in $L_{\beta}^{1}\left(\mathbb{R}_{+}^{d+1}\right)$

$$
\begin{gathered}
\mathcal{F}_{W}(f)(y)=\int_{\mathbb{R}_{+}^{d+1}} f(x) \Lambda(-x, y) \mathrm{d} \mu_{\beta}(x), \\
\text { for all } y \in \mathbb{R}_{+}^{d+1} .
\end{gathered}
$$

Some basic properties of this transform are the following:

1) For $f$ in $L_{\beta}^{1}\left(\mathbb{R}_{+}^{d+1}\right)$,

$$
\left\|\mathcal{F}_{W}(f)\right\|_{L_{\beta}^{\infty}\left(\mathbb{R}_{+}^{d+1}\right)} \leq\|f\|_{L_{\beta}^{1}\left(\mathbb{R}_{+}^{d+1}\right)} .
$$

2) For $f$ in $\mathcal{S}_{*}\left(\mathbb{R}^{d+1}\right)$ we have

$$
\mathcal{F}_{W}\left(\Delta_{\beta} f\right)(y)=-\|y\|^{2} \mathcal{F}_{W}(f)(y) \text {, for all } y \in \mathbb{R}_{+}^{d+1} \text {. }
$$

3) For all $f$ in $L_{\beta}^{1}\left(\mathbb{R}_{+}^{d+1}\right)$, if $\mathcal{F}_{W}(f)$ belongs to $L_{\beta}^{1}\left(\mathbb{R}_{+}^{d+1}\right)$, then

$$
f(y)=\int_{\mathbb{R}_{+}^{d+1}} \mathcal{F}_{W}(f)(x) \Lambda(x, y) \mathrm{d} \mu_{\beta}(x), \text { a.e. }
$$

4) For $f \in \mathcal{S}_{*}\left(\mathbb{R}^{d+1}\right)$, if we define

$$
\overline{\mathcal{F}_{W}}(f)(y)=\mathcal{F}_{W}(f)(-y),
$$

then

$$
\mathcal{F}_{W} \overline{\mathcal{F}_{W}}=\overline{\mathcal{F}_{W}} \mathcal{F}_{W}=\text { Id } .
$$

Proposition 1. 1) The Weinstein transform $\mathcal{F}_{W}$ is a topological isomorphism from $\mathcal{S}_{*}\left(\mathbb{R}^{d+1}\right)$ onto itself and for all $f$ in $\mathcal{S}_{*}\left(\mathbb{R}^{d+1}\right)$,

$$
\int_{\mathbb{R}_{+}^{d+1}}|f(x)|^{2} \mathrm{~d} \mu_{\beta}(x)=\int_{\mathbb{R}_{+}^{d+1}}\left|\mathcal{F}_{W}(f)(\xi)\right|^{2} \mathrm{~d} \mu_{\beta}(\xi) .
$$

2) In particular, the Weinstein transform $f \rightarrow \mathcal{F}_{W}(f)$ can be uniquely extended to an isometric isomorphism from $L_{\beta}^{2}\left(\mathbb{R}_{+}^{d+1}\right)$ onto itself.

The generalized translation operator $\tau_{x}, x \in \mathbb{R}_{+}^{d+1}$, associated with the operator $\Delta_{\beta}$ is defined by

$$
\forall y \in \mathbb{R}_{+}^{d+1}, \tau_{x} f(y)=\frac{\Gamma(\beta+1)}{\sqrt{\pi} \Gamma\left(\beta+\frac{1}{2}\right)} \int_{0}^{\pi} f\left(x^{\prime}+y^{\prime}, \sqrt{x_{d+1}^{2}+y_{d+1}^{2}+2 x_{d+1} y_{d+1} \cos \theta}\right)(\sin \theta)^{2 \beta} \mathrm{d} \theta
$$


where $f \in C_{*}\left(\mathbb{R}^{d+1}\right)$.

By using the Weinstein kernel, we can also define a generalized translation. For a function $f \in L_{\beta}^{2}\left(\mathbb{R}_{+}^{d+1}\right)$ and $y \in \mathbb{R}_{+}^{d+1}$ the generalized translation $\tau_{y} f$ is defined by the following relation:

$$
\mathcal{F}_{W}\left(\tau_{y} f\right)(x)=\Lambda(x, y) \mathcal{F}_{W}(f)(x) .
$$

For example, for $t>0$, we see that

$$
\tau_{y}\left(e^{-t \mid \xi \|^{2}}\right)(x)=e^{-t\left(\|x\|^{2}+\|y\|^{2}\right)} \Lambda(-i 2 t y, x) .
$$

By using the generalized translation, we define the generalized convolution product $f *_{\beta} g$ of functions $f, g \in L_{\beta}^{1}\left(\mathbb{R}_{+}^{d+1}\right)$ as follows:

$$
f *_{\beta} g(x)=\int_{\mathbb{R}_{+}^{d+1}} \tau_{x} f\left(-y^{\prime}, y_{d+1}\right) g(y) \mathrm{d} \mu_{\beta}(y) .
$$

This convolution is commutative and associative and satisfies the following propositions:

Proposition 2. 1) For all $f, g \in L_{\beta}^{1}\left(\mathbb{R}_{+}^{d+1}\right), \quad f *_{\beta} g$ belongs to $L_{\beta}^{1}\left(\mathbb{R}_{+}^{d+1}\right)$ and

$$
\mathcal{F}_{W}\left(f *_{\beta} g\right)=\mathcal{F}_{W}(f) \mathcal{F}_{W}(g) \text {. }
$$

2) Let $1 \leq p, q, r \leq \infty$ such that $\frac{1}{p}+\frac{1}{q}-\frac{1}{r}=1$. If $f \in L_{\beta}^{p}\left(\mathbb{R}_{+}^{d+1}\right)$ and $g \in L_{\beta}^{q}\left(\mathbb{R}_{+}^{d+1}\right)$, then

$f *_{\beta} g \in L_{\beta}^{r}\left(\mathbb{R}_{+}^{d+1}\right)$ and

$$
\left\|f *_{\beta} g\right\|_{L_{\beta}^{r}\left(\mathbb{R}_{+}^{d+1}\right)} \leq\|f\|_{L_{\beta}^{p}\left(\mathbb{R}_{+}^{d+1}\right)}\|g\|_{L_{\beta}^{q}\left(\mathbb{R}_{+}^{d+1}\right)} .
$$

Proposition 3. Let $f, g \in L_{\beta}^{2}\left(\mathbb{R}_{+}^{d+1}\right)$. Then $f *_{\beta} g \in L_{\beta}^{2}\left(\mathbb{R}_{+}^{d+1}\right)$ if and only if $\mathcal{F}_{W}(f) \mathcal{F}_{W}(g)$ belongs to $L_{\beta}^{2}\left(\mathbb{R}_{+}^{d+1}\right)$, and in this case we have

$$
\mathcal{F}_{W}\left(f *_{\beta} g\right)=\mathcal{F}_{W}(f) \mathcal{F}_{W}(g) .
$$

An immediate consequence of Proposition 3 and the Plancherel formula that will be used in the next section is the following. have

Proposition 4 Let $f$ and $g$ be in $L_{\beta}^{2}\left(\mathbb{R}_{+}^{d+1}\right)$. Then, we

$$
\begin{aligned}
& \int_{\mathbb{R}_{+}^{d+1}}\left|f *_{\beta} g(x)\right|^{2} \mathrm{~d} \mu_{\beta}(x) \\
& =\int_{\mathbb{R}_{+}^{d+1}}\left|\mathcal{F}_{W}(f)(\xi)\right|^{2}\left|\mathcal{F}_{W}(g)(\xi)\right|^{2} \mathrm{~d} \mu_{\beta}(\xi)
\end{aligned}
$$

where both sides are finite or infinite.

\section{The Continuous Weinstein Gabor Transform}

Notations. We denote by:

$X_{\beta}^{p}, p \in[1, \infty]$ the space of measurable functions $f$ on $\mathbb{R}_{+}^{d+1} \times \mathbb{R}_{+}^{d+1}$ with respect to the measure $\mathrm{d} \omega_{\beta}(x, y)=\mathrm{d} \mu_{\beta}(x) \mathrm{d} \mu_{\beta}(y)$ such that

$$
\begin{gathered}
\|f\|_{p, \omega_{\beta}}:=\left(\int_{\mathbb{R}_{+}^{d+1} \times \mathbb{R}_{+}^{d+1}}|f(x, y)|^{p} \mathrm{~d} \omega_{\beta}(x, y)\right)^{\frac{1}{p}}<\infty, \\
\quad 1 \leq p<\infty \\
\|f\|_{\infty, \omega_{\beta}}:=\underset{x, y \in \mathbb{R}_{+}^{d+1}}{\operatorname{essup}}|f(x, y)|<\infty .
\end{gathered}
$$

Definition 1. For any function $g$ in $L_{\beta}^{2}\left(\mathbb{R}_{+}^{d+1}\right)$ and any $v \in \mathbb{R}_{+}^{d+1}$, we define the modulation of $g$ by $v$ as:

$$
\mathcal{M}_{v} g:=g_{v}:=\mathcal{F}_{W}\left(\sqrt{\tau_{v}\left(|g|^{2}\right)}\right),
$$

where $\tau_{-y}, \quad y \in \mathbb{R}_{+}^{d+1}$, are the Weinstein translation operators given by (13).

Remark 1. For $g$ in $L_{\beta}^{2}\left(\mathbb{R}_{+}^{d+1}\right)$, we have

$$
\left\|g_{v}\right\|_{L_{\beta}^{2}\left(\mathbb{R}_{+}^{d+1}\right)}=\|g\|_{L_{\beta}^{2}\left(\mathbb{R}_{+}^{d+1}\right)} .
$$

We consider the family $g_{v, y}, v, y \in \mathbb{R}_{+}^{d+1}$ defined by

$$
g_{v, y}(x)=\tau_{-y} g_{v}(x), x \in \mathbb{R}_{+}^{d+1},
$$

Definition 2. Let $g$ be in $L_{\beta}^{2}\left(\mathbb{R}_{+}^{d+1}\right)$. For a function $f$ in $L_{\beta}^{2}\left(\mathbb{R}_{+}^{d+1}\right)$ we define its continuous Weinstein Gabor transform by

$$
\mathcal{G}_{g} f(y, v):=\int_{\mathbb{R}_{+}^{d+1}} f(x) \overline{g_{v, y}(x)} \mathrm{d} \mu_{\beta}(x),
$$

which can also be written in the form

$$
\mathcal{G}_{g} f(y, v):=f *_{\beta} \overline{\breve{g}}_{v}(y)
$$

where $\breve{h}(x)=\overline{h(-x)}$.

Theorem 1. ( $L_{\beta}^{2}$ inversion formula).

Let $g$ be in $\left(L_{\beta}^{2}\left(\mathbb{R}_{+}^{d+1}\right) \cap L_{\beta}^{\infty}\left(\mathbb{R}_{+}^{d+1}\right)\right) \backslash\{0\}$. Then, for any function $f$ in $L_{\beta}^{2}\left(\mathbb{R}_{+}^{d+1}\right)$, we have

$$
f_{n}(x)=\int_{B_{+}^{d+1}(0, n)} \int_{\mathbb{R}_{+}^{d+1}} \mathcal{G}_{g}(f)(y, v) \tau_{-y} g_{v}(x) \mathrm{d} \omega_{\beta}(v, y)
$$

in $L_{\beta}^{2}\left(\mathbb{R}_{+}^{d+1}\right)$, where

$$
\mathrm{d} \omega_{\beta}(v, y):=\mathrm{d} \mu_{\beta}(y) \mathrm{d} \mu_{\beta}(v)
$$

and satisfies

$$
\lim _{n \rightarrow \infty}\left\|f-f_{n}\right\|_{L_{\beta}^{2}\left(\mathbb{R}_{+}^{d+1}\right)}=0 .
$$

For proof this theorem we need the following Lemmas.

Lemma 1. Let $g$ be as above. For any positive integer $n$ define the two functions

$$
\begin{aligned}
& G_{n}(x) \\
& :=\int_{B_{+}^{d+1}(0, n)} \int_{\mathbb{R}_{+}^{d+1}} \Lambda(\xi, x)\left|\mathcal{F}_{W}\left(g_{v}\right)(\xi)\right|^{2} \mathrm{~d} \mu_{\beta}(v) \mathrm{d} \mu_{\beta}(\xi),
\end{aligned}
$$


for $x \in \mathbb{R}_{+}^{d+1}$, and

$$
H_{n}(\xi):=\int_{B_{+}^{d+1}(0, n)}\left|\mathcal{F}_{W}\left(g_{v}\right)(\xi)\right|^{2} \mathrm{~d} \mu_{\beta}(v),
$$

for $\xi \in \mathbb{R}_{+}^{d+1}$. Then

$$
\begin{gathered}
G_{n} \in L_{\beta}^{2}\left(\mathbb{R}_{+}^{d+1}\right), H_{n} \in L_{\beta}^{1}\left(\mathbb{R}_{+}^{d+1}\right) \cap L_{\beta}^{\infty}\left(\mathbb{R}_{+}^{d+1}\right), \\
\text { and } \mathcal{F}_{W}\left(G_{n}\right)=H_{n} .
\end{gathered}
$$

Proof. Using the Cauchy-Schwartz inequality we obtain

$$
\begin{aligned}
\left|G_{n}(x)\right|^{2} & \leq\left.\left. C\left(\int_{B_{+}^{d+1}(0, n)} \mathrm{d} \mu_{\beta}(v)\right) \int_{B_{+}^{d+1}(0, n)}\left|\int_{\mathbb{R}_{+}^{d+1}} \Lambda(\xi, x)\right| \mathcal{F}_{W}\left(g_{v}\right)(\xi)\right|^{2} \mathrm{~d} \mu_{\beta}(\xi)\right|^{2} \mathrm{~d} \mu_{\beta}(v) \\
& \leq\left.\left. C \int_{B_{+}^{d+1}(0, n)}\left|\int_{\mathbb{R}_{+}^{d+1}} \Lambda(\xi, x)\right| \mathcal{F}_{W}\left(g_{v}\right)(\xi)\right|^{2} \mathrm{~d} \mu_{\beta}(\xi)\right|^{2} \mathrm{~d} \mu_{\beta}(v) .
\end{aligned}
$$

Therefore by Fubini theorem, the inversion theorem, the Plancherel formula and Proposition 3

$$
\begin{aligned}
\int_{\mathbb{R}_{+}^{d+1}}\left|G_{n}(x)\right|^{2} \mathrm{~d} \mu_{\beta}(x) & \leq\left.\left. C \int_{B_{+}^{d+1}(0, n)} \int_{\mathbb{R}_{+}^{d+1}}\left|\int_{\mathbb{R}_{+}^{d+1}} \Lambda(\xi, x)\right| \mathcal{F}_{W}\left(g_{v}\right)(\xi)\right|^{2} \mathrm{~d} \mu_{\beta}(\xi)\right|^{2} \mathrm{~d} \mu_{\beta}(v) \mathrm{d} \mu_{\beta}(x) \\
& \leq C \int_{B_{+}^{d+1}(0, n)} \int_{\mathbb{R}_{+}^{d+1}}\left|\mathcal{F}_{W}^{-1}\left(\left|\mathcal{F}_{W}(g)\right|^{2}\right)(x)\right|^{2} \mathrm{~d} \mu_{\beta}(x) \mathrm{d} \mu_{\beta}(v) \\
& \leq\left.\left. C \int_{B_{+}^{d+1}(0, n)} \int_{\mathbb{R}_{+}^{d+1}}\left|\tau_{v}\right| g\right|^{2}(\xi)\right|^{2} \mathrm{~d} \mu_{\beta}(v) \mathrm{d} \mu_{\beta}(\xi) \\
& \leq\left. C \int_{B_{+}^{d+1}(0, n)}\left|\tau_{v}\right| g\right|^{2}\left\|_{L_{\beta}^{1}\left(\mathbb{R}_{+}^{d+1}\right)}\right\| \tau_{v}|g|^{2} \|_{L_{\beta}^{\infty}\left(\mathbb{R}_{+}^{d+1}\right)} \mathrm{d} \mu_{\beta}(v) \\
& \leq C \int_{B_{+}^{d+1}(0, n)}\left\|\tau_{v}|g|^{2}\right\|_{L_{\beta}^{\infty}\left(\mathbb{R}_{+}^{d+1}\right)} \mathrm{d} \mu_{\beta}(v)<\infty .
\end{aligned}
$$

That $H_{n} \in L_{\beta}^{1}\left(\mathbb{R}_{+}^{d+1}\right) \cap L_{\beta}^{\infty}\left(\mathbb{R}_{+}^{d+1}\right)$ is easily checked. Finally, using Fubinis theorem we obtain

$$
\begin{aligned}
\mathcal{F}_{W}^{-1}\left(H_{n}\right)(y) & =\int_{\mathbb{R}_{+}^{d+1}} H_{n}(\xi) \Lambda(\xi, y) \mathrm{d} \mu_{\beta}(\xi) \\
& =\int_{\mathbb{R}_{+}^{d+1}} \Lambda(\xi, y) \int_{B_{+}^{d+1}(0, n)}\left|\mathcal{F}_{W}\left(g_{v}\right)(\xi)\right|^{2} \mathrm{~d} \mu_{\beta}(v) \mathrm{d} \mu_{\beta}(\xi) \\
& =\int_{B_{+}^{d+1}(0, n)} \int_{\mathbb{R}_{+}^{d+1}} \Lambda(\xi, y)\left|\mathcal{F}_{W}\left(g_{v}\right)(\xi)\right|^{2} \mathrm{~d} \mu_{\beta}(v) \mathrm{d} \mu_{\beta}(\xi)=G_{n}(y) .
\end{aligned}
$$

Lemma 2. Let $g$ be as above. For any positive integer $n$ the function

$$
\begin{aligned}
& G_{n}(x) \\
& :=\int_{B_{+}^{d+1}(0, n)} \int_{\mathbb{R}_{+}^{d+1}} \Lambda(\xi, x)\left|\mathcal{F}_{W}\left(g_{v}\right)(\xi)\right|^{2} \mathrm{~d} \mu_{\beta}(\xi) \mathrm{d} \mu_{\beta}(v),
\end{aligned}
$$

can be written

$$
G_{n}(x)=\int_{B_{+}^{d+1}(0, n)} \breve{g}_{v} *_{\beta} g_{v}(x) \mathrm{d} \mu_{\beta}(v) .
$$

Proof. From Proposition 3 we have

$$
\begin{aligned}
& G_{n}(x) \\
& =\int_{B_{+}^{d+1}(0, n)} \int_{\mathbb{R}_{+}^{d+1}} \Lambda(\xi, x)\left|\mathcal{F}_{W}\left(g_{v}\right)(\xi)\right|^{2} \mathrm{~d} \mu_{\beta}(v) \mathrm{d} \mu_{\beta}(\xi) \\
& =\int_{B_{+}^{d+1}(0, n)} \mathcal{F}_{W}^{-1}\left(\left|\mathcal{F}_{W}\left(g_{v}\right)\right|^{2}\right)(x) \mathrm{d} \mu_{\beta}(v) \\
& =\int_{B_{+}^{d+1}(0, n)}\left(\breve{g}_{v} *_{\beta} g_{v}(x)\right) \mathrm{d} \mu_{\beta}(v) .
\end{aligned}
$$

Lemma 3. Let $g$ be in $\left(L_{\beta}^{2}\left(\mathbb{R}_{+}^{d+1}\right) \cap L_{\beta}^{\infty}\left(\mathbb{R}_{+}^{d+1}\right)\right) \backslash\{0\}$. Then, for any function $f$ in $L_{\beta}^{2}\left(\mathbb{R}_{+}^{d+1}\right)$, we have

$$
f_{n}=G_{n} *_{\beta} f \text {. }
$$

Proof. We have

$$
\begin{aligned}
& f_{n}(x) \\
& =\int_{B_{+}^{d+1}(0, n)} \int_{\mathbb{R}_{+}^{d+1}} \mathcal{G}_{g}(f)(y, v) \tau_{-y} g_{v}(x) \mathrm{d} \omega_{\beta}(v, y) \\
& =\int_{B_{+}^{d+1}(0, n)}\left(\mathcal{G}_{g}(f)(., v) *_{\beta} g_{v}\right)(x) \mathrm{d} \mu_{\beta}(v) \\
& =\int_{B_{+}^{d+1}(0, n)} f *_{\beta} \breve{g}_{v} *_{\beta} g_{v}(x) \mathrm{d} \mu_{\beta}(v) \\
& =\int_{B_{+}^{d+1}(0, n)} \int_{\mathbb{R}_{+}^{d+1}} \tau_{x} f(y) \breve{g}_{v} *_{\beta} g_{v}(-y) \mathrm{d} \omega_{\beta}(v, y) \\
& =\int_{\mathbb{R}_{+}^{d+1}} \tau_{x} f(y)\left(\int_{B_{+}^{d+1}(0, n)} \breve{g}_{v} *_{\beta} g_{v}(-y) \mathrm{d} \mu_{\beta}(v)\right) \mathrm{d} \mu_{\beta}(y) \\
& =\int_{\mathbb{R}_{+}^{d+1}} \tau_{x} f(y) G_{n}(-y) \mathrm{d} \mu_{\beta}(y) \\
& =f *_{\beta} G_{n}(x) .
\end{aligned}
$$

On the follow we justify the use of Fubinis theorem in the last sequence of equalities observe that

$$
\begin{aligned}
& \left|\int_{B_{+}^{d+1}(0, n)} \int_{\mathbb{R}_{+}^{d+1}} \tau_{x} f(y) \breve{g}_{v} *_{\beta} g_{v}(-y) \mathrm{d} \omega_{\beta}(v, y)\right| \\
& \leq \int_{B_{+}^{d+1}(0, n)}\left|f *_{\beta} \breve{g}_{v} *_{\beta} g_{v}(x)\right| \mathrm{d} \mu_{\beta}(v) .
\end{aligned}
$$

Now, using Proposition 3 and hypothesis on $g$ we see 
that $\breve{g}_{v} *_{\beta} g_{v} \in L_{\beta}^{2}\left(\mathbb{R}_{+}^{d+1}\right)$. Next using Young's inequality and Parseval theorem we obtain

$$
\begin{aligned}
& \left\|f *_{\beta} \breve{g}_{v} *_{\beta} g_{v}\right\|_{L_{\beta}^{\infty}\left(\mathbb{R}_{+}^{d+1}\right)} \\
& \leq\|f\|_{L_{\beta}^{2}\left(\mathbb{R}_{+}^{d+1}\right)}\left\|\breve{g}_{v} *_{\beta} g_{v}\right\|_{L_{\beta}^{2}\left(\mathbb{R}_{+}^{d+1}\right)} \leq C\|f\|_{L_{\beta}^{2}\left(\mathbb{R}_{+}^{d+1}\right)}\|g\|_{L_{\beta}^{2}\left(\mathbb{R}_{+}^{d+1}\right)}\|g\|_{L_{\beta}^{\infty}\left(\mathbb{R}_{+}^{d+1}\right)}
\end{aligned}
$$

and

$$
\begin{aligned}
& \int_{B_{+}^{d+1}(0, n)}\left|f *_{\beta} \breve{g}_{v} *_{\beta} g_{v}(x)\right| \mathrm{d} \mu_{\beta}(v) \\
& \leq C\left(\int_{B_{+}^{d+1}(0, n)} \mathrm{d} \mu_{\beta}(v)\|f\|_{L_{\beta}^{2}\left(\mathbb{R}_{+}^{d+1}\right)}\|g\|_{L_{\beta}^{2}\left(\mathbb{R}_{+}^{d+1}\right)}\|g\|_{L_{\beta}^{\infty}\left(\mathbb{R}_{+}^{d+1}\right)} .\right.
\end{aligned}
$$

The proof is complete.

Proof. of Theorem 1.

It follows from Proposition 3 and Lemma 1 that $f_{n} \in L_{\beta}^{2}\left(\mathbb{R}_{+}^{d+1}\right)$ and

$$
\mathcal{F}_{W}\left(f_{n}\right)=H_{n} \mathcal{F}_{W}(f) .
$$

By this, the Plancherel formula, the fact that $H_{n} \rightarrow 1$ pointwise as $n \rightarrow \infty$, and the dominated convergence theorem, it follows that

$$
\begin{aligned}
& \left\|f-f_{n}\right\|_{L_{\beta}^{2}\left(\mathbb{R}_{+}^{d+1}\right)}^{2} \\
& =\int_{\mathbb{R}_{+}^{d+1}}\left|\mathcal{F}_{W}(f)(\xi)-H_{n}(\xi) \mathcal{F}_{W}(f)(\xi)\right|^{2} \mathrm{~d} \mu_{\beta}(\xi) \\
& =\int_{\mathbb{R}_{+}^{d+1}}\left|\mathcal{F}_{W}(f)(\xi)\left(1-H_{n}(\xi)\right)\right|^{2} \mathrm{~d} \mu_{\beta}(\xi) \rightarrow 0
\end{aligned}
$$

as $n \rightarrow \infty$ which achieves the proof.

Proposition 5. For $f$ in $L_{\beta}^{2}\left(\mathbb{R}_{+}^{d+1}\right)$ and $g$ in $L_{\beta}^{2}\left(\mathbb{R}_{+}^{d+1}\right)$ we have

$$
\left\|\mathcal{G}_{g} f\right\|_{\infty, \omega_{\beta}} \leq\|f\|_{L_{\beta}^{2}\left(\mathbb{R}_{+}^{d+1}\right)}\|g\|_{L_{\beta}^{2}\left(\mathbb{R}_{+}^{d+1}\right)} .
$$

Proposition 6. (Plancherel formula).

Let $g$ be in $L_{\beta}^{2}\left(\mathbb{R}_{+}^{d+1}\right)$. Then, for all $f$ in $L_{\beta}^{2}\left(\mathbb{R}_{+}^{d+1}\right)$, we have

$$
\left\|\mathcal{G}_{g} f\right\|_{2, \omega_{\beta}}=\|g\|_{L_{\beta}^{2}\left(\mathbb{R}_{+}^{d+1}\right)}\|f\|_{L_{\beta}^{2}\left(\mathbb{R}_{+}^{d+1}\right)} .
$$

Proof. Using relation (18), Fubini's theorem and Plancherel's formula for the Weinstein transform, we have

$$
\begin{aligned}
& \int_{\mathbb{R}_{+}^{d+1}} \int_{\mathbb{R}_{+}^{d+1}}\left|f *_{\beta} \breve{g}_{v}\right|^{2}(y) \mathrm{d} \mu_{\beta}(y) \mathrm{d} \mu_{\beta}(v) \\
& =\int_{\mathbb{R}_{+}^{d+1}} \int_{\mathbb{R}_{+}^{d+1}}\left|\mathcal{F}_{W}(f)(\xi)\right|^{2}\left|\mathcal{F}_{W}\left(g_{v}\right)(\xi)\right|^{2} \mathrm{~d} \mu_{\beta}(\xi) \mathrm{d} \mu_{\beta}(v) \\
& =\int_{\mathbb{R}_{+}^{d+1}} \int_{\mathbb{R}_{+}^{d+1}}\left|\mathcal{F}_{W}(f)(\xi)\right|^{2} \tau_{v}\left(|g|^{2}\right)(\xi) \mathrm{d} \mu_{\beta}(\xi) \mathrm{d} \mu_{\beta}(v) \\
& =\int_{\mathbb{R}_{+}^{d+1}} \int_{\mathbb{R}_{+}^{d+1}}\left|\mathcal{F}_{W}(f)(\xi)\right|^{2} \tau_{\xi}\left(|g|^{2}\right)(v) \mathrm{d} \mu_{\beta}(v) \mathrm{d} \mu_{\beta}(\xi) \\
& =\|f\|_{L_{\beta}^{2}\left(\mathbb{R}_{+}^{d+1}\right)}^{2}\|g\|_{L_{\beta}^{2}\left(\mathbb{R}_{+}^{d+1}\right)}^{2}
\end{aligned}
$$

As in the classical case, the continuous Weinstein Ga- bor transform preserves the orthogonality relation. However, we have the following result.

Corollary 1. Let $g$ be in $L_{\beta}^{2}\left(\mathbb{R}_{+}^{d+1}\right)$. Then, for all $f, h$ in $L_{\beta}^{2}\left(\mathbb{R}_{+}^{d+1}\right)$, we have

$$
\begin{aligned}
& \int_{\mathbb{R}_{+}^{d+1}} \int_{\mathbb{R}_{+}^{d+1}} \mathcal{G}_{g} f(y, v) \overline{\mathcal{G}_{g} h(y, v)} \mathrm{d} \mu_{\beta}(y) \mathrm{d} \mu_{\beta}(v) \\
& =\|g\|_{L_{\beta}^{2}\left(\mathbb{R}_{+}^{d+1}\right)}^{2} \int_{\mathbb{R}_{+}^{d+1}} f(x) \overline{h(x)} \mathrm{d} \mu_{\beta}(x) .
\end{aligned}
$$

In what follows, we show the weak uncertainty princeple for the continuous Weinstein Gabor transform.

Proposition 7. Let $g$ be in $L_{\beta}^{2}\left(\mathbb{R}_{+}^{d+1}\right)$ such that $\|g\|_{L_{\beta}^{2}\left(\mathbb{R}_{+}^{d+1}\right)}=1$ and $f$ in $L_{\beta}^{2}\left(\mathbb{R}_{+}^{d+1}\right)$. Suppose that $\|f\|_{L_{\beta}^{2}\left(\mathbb{R}_{+}^{d+1}\right)}=1$ Then, for $U \subset \mathbb{R}_{+}^{d+1} \times \mathbb{R}_{+}^{d+1}$ and $\varepsilon>0$ satisfying

$$
\iint_{U}\left|\mathcal{G}_{g} f(y, v)\right|^{2} \mathrm{~d} \omega_{\beta}(y, v) \geq 1-\varepsilon
$$

we have

$$
\omega_{\beta}(U) \geq(1-\varepsilon)
$$

where

$$
\omega_{\beta}(U):=\int_{U} \mathrm{~d} \omega_{\beta}(x, y)<\infty .
$$

Proof. From the relation (24) we deduce that

$$
\left\|\mathcal{G}_{g} f\right\|_{\infty, \omega_{\beta}} \leq 1
$$

Thus,

$$
\begin{aligned}
1-\varepsilon & \leq \iint_{U}\left|\mathcal{G}_{g} f(y, v)\right|^{2} \mathrm{~d} \omega_{\beta}(y, v) \\
& \leq\left\|\mathcal{G}_{g} f\right\|_{\infty, \omega_{\beta}}^{2} \omega_{\beta}(U) \leq \omega_{\beta}(U) .
\end{aligned}
$$

This achieves the proof.

Proposition 8. Let $f$ be in $L_{\beta}^{2}\left(\mathbb{R}_{+}^{d+1}\right), g$ in $L_{\beta}^{2}\left(\mathbb{R}_{+}^{d+1}\right)$ such that $\|g\|_{L_{\beta}^{2}\left(\mathbb{R}_{+}^{d+1}\right)}=1$ and $p \in[2, \infty)$. Then,

$$
\int_{\mathbb{R}_{+}^{d+1} \times \mathbb{R}_{+}^{d+1}}\left|\mathcal{G}_{g} f(y, v)\right|^{p} \mathrm{~d} \omega_{\beta}(y, v) \leq\|f\|_{L_{\beta}^{2}\left(\mathbb{R}_{+}^{d+1}\right)}^{p} .
$$

Proof. Using Proposition 5 and Proposition 6 the result follows by applying the Riesz-Thorin interpolation theorem.

\section{Uncertainty Principles of Heisenberg Type}

In this section we will to prove the Heisenberg inequality for the generalized continuous Gabor transform.

Proposition 9. (Uncertainty principle of Heisenberg type for $\mathcal{F}_{W}$ ).

Let $f$ be in $L_{\beta}^{2}\left(\mathbb{R}_{+}^{d+1}\right)$, the following inequality holds 


$$
\left(\int_{\mathbb{R}_{+}^{d+1}}\|x\|^{2}\left|\mathcal{F}_{W}(f)(x)\right|^{2} \mathrm{~d} \mu_{\beta}(x)\right)^{\frac{1}{2}}\left(\int_{\mathbb{R}^{d}}\|y\|^{2}|f(y)|^{2} \mathrm{~d} \mu_{\beta}(y)\right)^{\frac{1}{2}} \geq\left(\beta+1+\frac{d}{2}\right)\|f\|_{L_{\beta}^{2}\left(\mathbb{R}_{+}^{d+1}\right)} \cdot
$$

Proof. We obtain the result by combining the Heisenberg inequalities for the classical Fourier transform and Fourier-Bessel transform.

Theorem 2. (Uncertainty principles of Heisenberg

\section{Type for $\mathcal{G}_{g}$ ).}

Let $g$ be in $L_{\beta}^{2}\left(\mathbb{R}_{+}^{d+1}\right)$. Then, for all $f$ in $L_{\beta}^{2}\left(\mathbb{R}_{+}^{d+1}\right)$, the following inequality holds

$$
\left(\int_{\mathbb{R}_{+}^{d+1}}\|x\|^{2}\left|\mathcal{F}_{W}(f)(x)\right|^{2} \mathrm{~d} \mu_{\beta}(x)\right)^{\frac{1}{2}}\left(\int_{\mathbb{R}_{+}^{d+1}} \int_{\mathbb{R}_{+}^{d+1}}\|y\|^{2}\left|\mathcal{G}_{g} f(y, v)\right|^{2} \mathrm{~d} \omega_{\beta}(y, v)\right)^{\frac{1}{2}} \geq\left(\beta+1+\frac{d}{2}\right)\|g\|_{L_{\beta}^{2}\left(\mathbb{R}_{+}^{d+1}\right)}\|f\|_{L_{\beta}^{2}\left(\mathbb{R}_{+}^{d+1}\right)} .
$$

Proof. Let us assume the non-trivial case that both integrals on the left hand side of (28) are finite. Fixing $v$ arbitrary, Heisenberg's inequality for Weinstein transform gives that

$$
\left(\int_{\mathbb{R}_{+}^{d+1}}\|y\|^{2}\left|\mathcal{F}_{W}\left(\mathcal{G}_{g} f(., v)\right)(y)\right|^{2} \mathrm{~d} \mu_{\beta}(y)\right)^{\frac{1}{2}}\left(\int_{\mathbb{R}_{+}^{d+1}}\|y\|^{2}\left|\mathcal{G}_{g} f(y, v)\right|^{2} \mathrm{~d} \mu_{\beta}(y)\right)^{\frac{1}{2}} \geq\left.\left(\beta+1+\frac{d}{2}\right) \int_{\mathbb{R}_{+}^{d+1}}|| \mathcal{G}_{g} f(y, v)\right|^{2} \mathrm{~d} \mu_{\beta}(y) .
$$

Integrating over $v$ and using Cauchy Schwartz inequality we obtain

$$
\begin{aligned}
& \left(\int_{\mathbb{R}_{+}^{d+1}} \int_{\mathbb{R}_{+}^{d+1}}\|y\|^{2}\left|\mathcal{F}_{W}\left(\mathcal{G}_{g} f(., v)\right)(y)\right|^{2} \mathrm{~d} \mu_{\beta}(y) \mathrm{d} \mu_{\beta}(v)\right)^{\frac{1}{2}}\left(\int_{\mathbb{R}_{+}^{d+1}} \int_{\mathbb{R}_{+}^{d+1}}\|y\|^{2}\left|\mathcal{G}_{g} f(y, v)\right|^{2} \mathrm{~d} \omega_{\beta}(y, v)\right)^{\frac{1}{2}} \\
& \geq\left(\beta+1+\frac{d}{2}\right) \int_{\mathbb{R}_{+}^{d+1}} \int_{\mathbb{R}_{+}^{d+1}}\left|\mathcal{G}_{g} f(y, v)\right|^{2} \mathrm{~d} \omega_{\beta}(y, v) .
\end{aligned}
$$

Thus, using the fact that

$$
\int_{\mathbb{R}_{+}^{d+1}} \int_{\mathbb{R}_{+}^{d+1}}\|y\|^{2}\left|\mathcal{F}_{W}\left(\mathcal{G}_{g} f(., v)\right)(y)\right|^{2} \mathrm{~d} \mu_{\beta}(y) \mathrm{d} \mu_{\beta}(v)=\|g\|_{L_{\beta}^{2}\left(\mathbb{R}_{+}^{d+1}\right)}^{2} \int_{\mathbb{R}_{+}^{d+1}}\|y\|^{2}\left|\mathcal{F}_{W}(f)(x)\right|^{2} \mathrm{~d} \mu_{\beta}(y),
$$

we obtain

$$
\begin{aligned}
& \|g\|_{L_{\beta}^{2}\left(\mathbb{R}_{+}^{d+1}\right)}\left(\int_{\mathbb{R}_{+}^{d+1}}\|x\|^{2}\left|\mathcal{F}_{W}(f)(x)\right|^{2} \mathrm{~d} \mu_{\beta}(x)\right)^{\frac{1}{2}}\left(\int_{\mathbb{R}_{+}^{d+1}} \int_{\mathbb{R}_{+}^{d+1}}\|y\|^{2}\left|\mathcal{G}_{g} f(y, v)\right|^{2} \mathrm{~d} \omega_{\beta}(y, v)\right)^{\frac{1}{2}} \\
& \geq\left(\beta+1+\frac{d}{2}\right) \int_{\mathbb{R}_{+}^{d+1}} \int_{\mathbb{R}_{+}^{d+1}}\left|\mathcal{G}_{g} f(y, v)\right|^{2} \mathrm{~d} \omega_{\beta}(y, v)=\left(\beta+1+\frac{d}{2}\right)\|g\|_{L_{\beta}^{2}(\mathbb{R})}^{2}\|f\|_{L_{\beta}^{2}(\mathbb{R})}^{2} .
\end{aligned}
$$

This proves the result.

\section{Reproducing Kernel}

Corollary 2. (Reproducing Kernel). Let $g$ be in

$$
\left(L_{\beta}^{2}\left(\mathbb{R}_{+}^{d+1}\right) \cap L_{\beta}^{\infty}\left(\mathbb{R}_{+}^{d+1}\right)\right) \backslash\{0\} \text {. Then, } \mathcal{G}_{g}\left(L_{\beta}^{2}\left(\mathbb{R}_{+}^{d+1}\right)\right) \text { is }
$$
a reproducing kernel Hilbert space in $X_{\beta}^{2}$ with kernel function

$$
\mathcal{W}_{g}\left(y^{\prime}, v^{\prime} ; y, v\right):=\frac{1}{\|g\|_{L_{\beta}^{2}\left(\mathbb{R}_{+}^{d+1}\right)}^{2}} \int_{\mathbb{R}_{+}^{d+1}} \tau_{y} \breve{g}_{v}(x) \tau_{y^{\prime}} g_{v^{\prime}}(x) \mathrm{d} \mu_{\beta}(x):=\frac{1}{\|g\|_{L_{\beta}^{2}\left(\mathbb{R}_{+}^{d+1}\right)}^{2}} \tau_{y} \breve{g}_{v} *_{\beta} \breve{g}_{v^{\prime}}\left(-y^{\prime}\right) .
$$

The kernel is pointwise bounded:

$$
\begin{array}{cc}
\left|\mathcal{W}_{g}\left(y^{\prime}, v^{\prime} ; y, v\right)\right| \leq 1 ; \text { for all }\left(y^{\prime}, v^{\prime}\right), & \mathcal{G}_{g} f(y, v)=\int_{\mathbb{R}_{+}^{d+1}} f(x) \overline{g_{v, y}(x)} \mathrm{d} \mu_{\beta}(x) . \\
(y, v) \in \mathbb{R}_{+}^{d+1} \times \mathbb{R}_{+}^{d+1} . & \text { Using the relation (26), we obtain } \\
\mathcal{G}_{g} f(y, v)=\frac{1}{\|g\|_{L_{\beta}^{2}\left(\mathbb{R}_{+}^{d+1}\right)}^{2}} \int_{\mathbb{R}_{+}^{d+1}} \int_{\mathbb{R}_{+}^{d+1}} \mathcal{G}_{g} f\left(x, v^{\prime}\right) \overline{\mathcal{G}_{g}\left(g_{v, y}\right)\left(x^{\prime}, v^{\prime}\right)} \mathrm{d} \mu_{\beta}(x) \cdot \mathrm{d} \mu_{\beta}(v)
\end{array}
$$

Proof. We have 
On the other hand using Proposition 3, one can easily see that for every $y, v, v^{\prime} \in \mathbb{R}_{+}^{d+1}$, the function

$$
\begin{aligned}
x & \mapsto \frac{1}{\|g\|_{L_{\beta}^{2}\left(\mathbb{R}_{+}^{d+1}\right)}^{2}} \overline{\mathcal{G}_{g}\left(g_{v, y}\right)\left(x, v^{\prime}\right)} \\
& =\frac{1}{\|g\|_{L_{\beta}^{2}\left(\mathbb{R}_{+}^{d+1}\right)}^{2}} \tau_{y} \breve{g}_{v} *_{\beta} \breve{g}_{v^{\prime}}(-x)
\end{aligned}
$$

belongs to $L_{\beta}^{2}\left(\mathbb{R}_{+}^{d+1}\right)$. Therefore, the result is obtained.

In the following theorem, we will show that the portion of the continuous Weinstein Gabor transform lying outside some sufficiently small set of finite measure cannot be arbitrarily too small. Then, in order to prove a concentration result of the continuous Weinstein Gabor transform, we need the following notations:

$P_{g}: X_{\beta}^{2} \rightarrow X_{\beta}^{2}$ the orthogonal projection from $X_{\beta}^{2}$ onto $\mathcal{G}_{g}\left(L_{\beta}^{2}\left(\mathbb{R}_{+}^{d+1}\right)\right)$.

$P_{U}: X_{\beta}^{2} \rightarrow X_{\beta}^{2}$ the orthogonal projection from $X_{\beta}^{2}$ onto the subspace of function supported in the subset $U \subset \mathbb{R}_{+}^{d+1} \times \mathbb{R}_{+}^{d+1}$ with $\omega_{\beta}(U)<\infty$.

We put

$$
\begin{array}{r}
\left\|P_{U} P_{g}\right\|=\sup \left\{\left\|P_{U} P_{g} v\right\|_{2, \omega_{\beta}}, v \in X_{\beta}^{2} ;\|v\|_{2, \omega_{\beta}}=1\right\} . \quad \text { (31) } \quad \begin{array}{l}
\text { bert space, then, from Sait } \\
\text { by }
\end{array} \\
P_{g} F(y, v)=\int_{\mathbb{R}_{+}^{d+1} \times \mathbb{R}_{+}^{d+1}} F\left(y^{\prime}, v^{\prime}\right) \mathcal{W}_{g}\left(y^{\prime}, v^{\prime} ; y, v\right) \mathrm{d} \mu_{\beta}\left(y^{\prime}, v^{\prime}\right),
\end{array}
$$

The essential result of this section is the following.

Theorem 3. (Concentration of $\mathcal{G}_{g} f$ in small sets) Let $g$ be in $\left(L_{\beta}^{2}\left(\mathbb{R}_{+}^{d+1}\right) \cap L_{\beta}^{\infty}\left(\mathbb{R}_{+}^{d+1}\right)\right) \backslash\{0\}$ and $U \subset \mathbb{R}_{+}^{d+1} \times \mathbb{R}_{+}^{d+1}$ with $\omega_{\beta}(U)<1$. Then, for all $f$ in $L_{\beta}^{2}\left(\mathbb{R}_{+}^{d+1}\right)$ we have

$$
\begin{aligned}
& \left\|\mathcal{G}_{g} f-\chi_{U} \mathcal{G}_{g} f\right\|_{2, \omega_{\beta}} \\
& \geq\left(1-\sqrt{\omega_{\beta}(U)}\right)\|g\|_{L_{\beta}^{2}\left(\mathbb{R}_{+}^{d+1}\right)}\|f\|_{L_{\beta}^{2}\left(\mathbb{R}_{+}^{d+1}\right)} .
\end{aligned}
$$

Proof. From the definition of $P_{U}$ and $P_{g}$ we have

$$
\left\|\mathcal{G}_{g} f-\chi_{U} \mathcal{G}_{g} f\right\|_{2, \omega_{\beta}}=\left\|\left(I-P_{U} P_{g}\right) \mathcal{G}_{g} f\right\|_{2, \omega_{\beta}} .
$$

Then, using the Proposition 6 we get

$$
\begin{gathered}
\left\|\mathcal{G}_{g} f-\chi_{U} \mathcal{G}_{g} f\right\|_{2, \omega_{\beta}} \geq\left\|\mathcal{G}_{g} f\right\|_{2, \omega_{\beta}}\left(1-\left\|P_{U} P_{g}\right\|\right) \\
\geq\|g\|_{L_{\beta}^{2}\left(\mathbb{R}_{+}^{d+1}\right)}\|f\|_{L_{\beta}^{2}\left(\mathbb{R}_{+}^{d+1}\right)}\left(1-\left\|P_{U} P_{g}\right\|\right) .
\end{gathered}
$$

As $P_{g}$ is a projection onto a reproducing kernel Hilbert space, then, from Saitoh [5], $P_{g}$ can be represented

with $\mathcal{W}_{g}$ defined by (29). Hence, for $F \in X_{\beta}^{2}$ arbitrary, we have

$$
P_{U} P_{g} F(y, v)=\int_{\mathbb{R}_{+}^{d+1} \times \mathbb{R}_{+}^{d+1}} \chi_{U}(y, v) F\left(y^{\prime}, v^{\prime}\right) \mathcal{G}_{g}\left(y^{\prime}, v^{\prime} ; y, v\right) \mathrm{d} \omega_{\beta}\left(y^{\prime}, v^{\prime}\right)
$$

and its Hilbert-Schmidt norm

$$
\left\|P_{U} P_{g}\right\|_{H S}=\left(\int_{\mathbb{R}_{+}^{2 d+2} \times \mathbb{R}_{+}^{2 d+2}}\left|\chi_{U}(y, v)\right|^{2}\left|\mathcal{W}_{g}\left(y^{\prime}, v^{\prime} ; y, v\right)\right|^{2} \mathrm{~d} \omega_{\beta}\left(y^{\prime}, v^{\prime}\right) \mathrm{d} \omega_{\beta}(y, v)\right)^{\frac{1}{2}} .
$$

By the Cauchy-Schwartz inequality we see that

$$
\left\|P_{U} P_{g}\right\|_{H S} \geq\left\|P_{U} P_{g}\right\| \text {. }
$$

On the other hand, from (29) and Fubini's theorem, it is easy to see that

$$
\begin{aligned}
\left\|P_{U} P_{g}\right\|_{H S} \leq \sqrt{\omega_{\beta}(U)} . \quad \text { (36) } \quad \text { Let } s \in \mathbb{R} . \text { We define the space } \\
H_{\beta}^{s}\left(\mathbb{R}_{+}^{d+1}\right):=\left\{f \in L_{\beta}^{2}\left(\mathbb{R}_{+}^{d+1}\right):\left(1+\|\xi\|^{2}\right)^{s / 2} \mathcal{F}_{W}(f) \in L_{\beta}^{2}\left(\mathbb{R}_{+}^{d+1}\right)\right\} .
\end{aligned}
$$

Thus, from the relations (34), (35) and (36) we obtain the result.

\section{Practical Real Inversion Formulas for $\mathcal{G}_{g}$}

Let $s \in \mathbb{R}$. We define the space $H_{\beta}^{s}\left(\mathbb{R}_{+}^{d+1}\right)$ by

The space $H_{\beta}^{s}\left(\mathbb{R}_{+}^{d+1}\right)$ provided with the inner product

$$
\langle f, g\rangle_{H_{\beta}^{s}\left(\mathbb{R}_{+}^{d+1}\right)}=\int_{\mathbb{R}_{+}^{d+1}}\left(1+\|\xi\|^{2}\right)^{s} \mathcal{F}_{W}(f)(\xi) \overline{\mathcal{F}_{W}(g)}(\xi) \mathrm{d} \mu_{\beta}(\xi),
$$

and the norm $\|f\|_{H_{\beta}^{s}\left(\mathbb{R}_{+}^{d+1}\right)}^{2}=\langle f, f\rangle_{H_{\beta}^{s}\left(\mathbb{R}_{+}^{d+1}\right)}$, is a Hilbert space.
Proposition 10. Let $g$ be a function in $L_{\beta}^{2}\left(\mathbb{R}_{+}^{d+1}\right) \cap L_{\beta}^{\infty}\left(\mathbb{R}_{+}^{d+1}\right)$, and $v \in \mathbb{R}_{+}^{d+1}$. The integral trans- 
form $\mathcal{G}_{g}(., v)$, is a bounded linear operator from $H_{\beta}^{s}\left(\mathbb{R}_{+}^{d+1}\right)$, s in $\mathbb{R}_{+}$, into $L_{\beta}^{2}\left(\mathbb{R}_{+}^{d+1}\right)$, and we have

$$
\left\|\mathcal{G}_{g} f(., v)\right\|_{L_{\beta}^{2}\left(\mathbb{R}_{+}^{d+1}\right)} \leq\|g\|_{L_{\beta}^{\infty}\left(\mathbb{R}_{+}^{d+1}\right)}\|f\|_{H_{\beta}^{s}\left(\mathbb{R}_{+}^{d+1}\right)} .
$$

Proof. We proceed as [6] we obtain the result.

Definition 3. Let $g$ be a function in

$L_{\beta}^{2}\left(\mathbb{R}_{+}^{d+1}\right) \cap L_{\beta}^{\infty}\left(\mathbb{R}_{+}^{d+1}\right)$. Let $r>0, \quad v \in \mathbb{R}_{+}^{d+1}$ and

$s \in \mathbb{R}_{+}$. We define the Hilbert space $H_{\beta}^{r, s}\left(\mathbb{R}_{+}^{d+1}\right)$ as the subspace of $H_{\beta}^{s}\left(\mathbb{R}_{+}^{d+1}\right)$ with the inner product:

$$
\begin{gathered}
\langle f, h\rangle_{H_{\beta}^{r, s}}=r\langle f, h\rangle_{H_{\beta}^{s}\left(\mathbb{R}_{+}^{d+1}\right)}+\left\langle\mathcal{G}_{g} f(., v), \mathcal{G}_{g} h(., v)\right\rangle_{L_{\beta}^{2}\left(\mathbb{R}_{+}^{d+1}\right)}, \\
f, h \in H_{\beta}^{s}\left(\mathbb{R}_{+}^{d+1}\right) .
\end{gathered}
$$

The norm associated to the inner product is defined by:

$$
\|f\|_{H_{\beta}^{r, s}}^{2}:=r\|f\|_{H_{\beta}^{s}\left(\mathbb{R}_{+}^{d+1}\right)}^{2}+\left\|\mathcal{G}_{g} f(., v)\right\|_{L_{\beta}^{2}\left(\mathbb{R}_{+}^{d+1}\right)}^{2} .
$$

We proceed as [6] we prove the following results.

Proposition 11. Let $g$ be a function in $L_{\beta}^{2}\left(\mathbb{R}_{+}^{d+1}\right) \cap L_{\beta}^{\infty}\left(\mathbb{R}_{+}^{d+1}\right)$. For $s \geq \beta+1+\frac{d}{2}$, the Hilbert space $H_{\beta}^{r, s}\left(\mathbb{R}_{+}^{d+1}\right)$ admits the following reproducing kernel:

$$
P_{g}(x, y)=\int_{\mathbb{R}_{+}^{d+1}} \frac{\Lambda(\xi, x) \Lambda(\xi, y) \mathrm{d} \mu_{\beta}(\xi)}{r\left(1+\|\xi\|^{2}\right)^{s}+\tau_{v}\left(|g|^{2}\right)(\xi)} .
$$

Theorem 4. Let $g$ be a function in

$$
L_{\beta}^{2}\left(\mathbb{R}_{+}^{d+1}\right) \cap L_{\beta}^{\infty}\left(\mathbb{R}_{+}^{d+1}\right) \text {. Let } s \geq \beta+1+\frac{d}{2} \text {. }
$$

1) For any $h$ in $L_{\beta}^{2}\left(\mathbb{R}_{+}^{d+1}\right)$ and for any $r>0$, the infinitum

$$
\inf _{f \in H_{\beta}^{s}\left(\mathbb{R}_{+}^{d+1}\right)}\left\{r\|f\|_{H_{\beta}^{s}\left(\mathbb{R}_{+}^{d+1}\right)}^{2}+\left\|h-\mathcal{G}_{g} f(., v)\right\|_{L_{\beta}^{2}\left(\mathbb{R}_{+}^{d+1}\right)}^{2}\right\}
$$

is attained by a unique function $f_{r, h}^{*}$ given by

$$
f_{r, h}^{*}(x)=\int_{\mathbb{R}_{+}^{d+1}} h(y) Q_{r}(x, y) \mathrm{d} \mu_{\beta}(y),
$$

where

$$
\begin{aligned}
& Q_{r}(x, y)=Q_{r, s}(x, y) \\
& =\int_{\mathbb{R}_{+}^{d+1}} \frac{\sqrt{\tau_{v}\left(|g|^{2}\right)(\xi)} \Lambda(\xi, x) \Lambda(\xi, y)}{r\left(1+\|\xi\|^{2}\right)^{s}+\tau_{v}\left(|g|^{2}\right)(\xi)} \mathrm{d} \mu_{\beta}(\xi) .
\end{aligned}
$$

2) Let $r, \delta>0$ and $h, h_{\delta}$ in $L_{\beta}^{2}\left(\mathbb{R}_{+}^{d+1}\right)$ such that

$$
\left\|h-h_{\delta}\right\|_{L_{\beta}^{2}\left(\mathbb{R}_{+}^{d+1}\right)} \leq \delta \text {. }
$$

Then

$$
\left\|f_{r, h}^{*}-f_{r, h_{\delta}}^{*}\right\|_{H_{\beta}^{s}\left(\mathbb{R}_{+}^{d+1}\right)} \leq \frac{\delta}{2 \sqrt{r}} .
$$

3) Let $r>0$. If $f$ is in $H_{\beta}^{s}\left(\mathbb{R}_{+}^{d+1}\right)$ and $h=\mathcal{G}_{g} f(., v)$. Then

$$
\left\|f_{r, h}^{*}-f\right\|_{H_{\beta}^{s}\left(\mathbb{R}_{+}^{d+1}\right)}^{2} \rightarrow 0 \text { as } r \rightarrow 0 .
$$

\section{REFERENCES}

[1] Z. B. Nahia and N. B. Salem, "Spherical Harmonics and Applications Associated with the Weinstein Operator," Potential Theory-ICPT 94, 1996, pp. 235-241.

[2] Z. B. Nahia and N. B. Salem, "On a Mean Value Property Associated with the Weinstein Operator," Potential Theory-ICPT 94, 1996, pp. 243-253.

[3] M. Brelot, "Equation de Weinstein et Potentiels de Marcel Riesz," Lecture Notes in Mathematics 681, Séminaire de Théorie de Potentiel Paris, No. 3, 1978, pp. 18-38.

[4] D. Gabor, "Theory of Communication," Proceedings of the Institute of Electrical Engineers, Vol. 93, No. 26, 1946, pp. 429-457.

[5] S. Saitoh, "Theory of Reproducing Kernels and Its Applications," Longman Scientific Technical, Harlow, 1988.

[6] H. Mejjaoli and N. Sraieb, "Gabor Transform in Quantum Calculus and Applications," Fractional Calculus and Applied Analysis, Vol. 12, No. 3, 2009, pp. 320-336. 